\title{
Can a Hospital's Analytics Capabilities Impact Patient Satisfaction? A Multi-Year Panel Study
}

\author{
Idris Adjerid \\ University of Notre Dame \\ iadjerid@nd.edu
}

\author{
Corey M. Angst \\ University of Notre Dame \\ cangst@nd.edu
}

\author{
Sarv Devaraj \\ University of Notre Dame \\ sdevaraj@nd.edu
}

\begin{abstract}
An empirical link between organizational performance and the IT necessary to enable data analytics capabilities has not yet been established. Drawing from organization information processing theory (OIPT), which argues that uncertainty and equivocality negatively impact organizational performance, we construct a model in which performance-measured as hospitals' patient satisfaction-is a function of clinical analytics capabilities, complexity, and concentration. Our argument is that clinical analytics is an uncertaintyreducing mechanism that directly impacts satisfaction. However, we propose a nuanced moderating role of complexity of patient cases and concentration (the mix of procedures performed in a hospital). We show that analytics capabilities increased patient satisfaction, but we also find evidence for the moderating role of complexity on the effect of analytics on satisfaction. The result for the moderating impact of concentration was not significant; however, our post-hoc analysis indicated that the moderating effect was present in larger hospitals.
\end{abstract}

\section{Introduction}

Organizations possess knowledge and use it to solve problems [1, 2], but certain situations are known to arise in which the knowledge required to complete a task is either not contained within the boundaries of the firm or not readily accessible in a useable format. The dramatic surge in recent years of both data analytics capabilities and organizational investment in these capabilities represents a significant step forward in bridging the gap between the knowledge required for organizations to perform optimally and what is readily available to them in practice. Despite there being little doubt that analytics is an IT-related phenomenon, surprisingly few studies have identified how or where analytics fits into IT research. In particular, whether and how the IT artifacts associated with analytics capabilities create value is particularly unclear [3-5]. We address this gap in the context of healthcare where investments in clinical and administrative technologies over the last two decades have resulted in hospitals with enormous stores of data that could yield potentially significant insights. This has spurred hospitals to increasingly adopt healthcare analytics (HA), or systems that extract and analyze data from other health IT systems to generate potentially useful clinical and operational insights (we provide examples of the operationalization of this later in the paper).

As in other industries, the potential benefits of HA in healthcare are substantial, but the link between the capabilities HA provides and hospital performance has not yet been theoretically explored or empirically validated. This is relevant since widespread or uniform gains in hospital performance as a result of HA adoption are by no means a foregone conclusion. A common theme of decades of IT value research (including work in healthcare) highlights that technology investments are rarely silver bullets where simply "turning on" the system is sufficient to realize promised gains. Rather, firms have to invest heavily in learning and training, exploit the complementarities, and reorganize process and workflows to accommodate IT investments [6-11]. In relation to healthcare specifically, a number of studies identify heterogeneous and nuanced effects of health IT adoption on health outcomes [e.g., 12, 13-15]. This body of work suggests that even if value does accrue from HA, it is unlikely that this value accrues homogenously across healthcare settings and hospitals.

In this paper, we use Organizational Information Processing Theory (OIPT) to investigate whether this relatively early-stage adoption of HA can improve hospital performance, and if so, what factors might moderate this effect. Specifically, we evaluate the impact of HA on hospitals' patient satisfaction, which is both of high importance to hospitals and could reasonably be impacted by adoption of HA. For example, patient satisfaction may be impacted as a function of better care- and also independent of care, 
if patients perceive that care supplemented by HA is more accurate or customized to them [16]. Aside from influencing and supplementing patient interactions, analytics may be applied to historic data on patient satisfaction to identify important predictors of high quality care [16].

OIPT is a particularly useful framework for investigating the impact of HA on patient satisfaction since it posits that reducing uncertainty —and when present, equivocality (ambiguity) —in decision making is key to improving performance. Along these lines, we contend that in healthcare environments generally replete with decision uncertainty [17, 18], HA will improve patient satisfaction, on average, because of its significant potential to resolve this uncertainty. Consistent with OIPT, we also argue that these effects may emerge unevenly because HA may not as effectively reduce equivocality in decision making, again when such equivocality is present. In addition to arguments in the extant literature suggesting that information systems are less useful for resolving equivocality, relative to uncertainty [19], current HA tools are still nascent and provide basic analytics capabilities [20]. More advanced HA capabilities (e.g., real-time generation of clinical insights and diagnoses) are both rare and hampered by lack of trust and acclimatization on the part of providers [21].

It follows then that clinical settings where resolution of equivocality is central to driving gains in patient satisfaction would benefit less from the adoption of healthcare analytics that reduce uncertainty in decision making. We consider two factors that moderate whether resolution of equivocality is central to driving gains in patient satisfaction: the clinical complexity of patients seen and hospitals' clinical concentration (the mix of procedures performed in a hospital, with higher concentration indicating that the hospital allocates a significant portion of its resources to a smaller number of clinical areas). We argue that these factors moderate the relationship between HA and patient satisfaction because hospitals with higher patient complexity and those that are more concentrated require substantive resolution of equivocality in decision making to achieve gains in patient satisfaction.

Our research makes a number of contributions. First, the literatures in operations, healthcare, policy, and information systems seem to be converging and showing that early investments in health IT can produce quality and efficiency gains-but often with the caveat that these benefits are nuanced [9, 22-25]. We contribute to what scholars [26] contend is the next frontier for this body of work, which is examining how data extracted and analyzed from these early IT investments in healthcare (e.g., EHRs) can improve the quality and efficiency of healthcare delivery [20]. Second, this work highlights a potentially useful theoretical framework for investigating the value of analytics capabilities more generally (i.e., beyond healthcare), and also identifies the types of factors that may moderate value (e.g., the nature of decision making challenges in a particular setting).

Our empirical approach leverages an eight-year panel dataset (2007-2014) which we created by combining several independent sources of data. We leverage between-hospital and across-time variation in both the adoption of HA and patient satisfaction to evaluate the impact of HA on patient satisfaction. We include hospital and time fixed effects, control for relevant observables, and perform an assortment of robustness checks to help address selection in hospital adoption of HA. Although the scale of our dataset precludes us from collecting detailed survey data, our work follows in the tradition of others who have used OIPT as a theoretical lens through which to examine uncertainty and equivocality resolution [19, 27, 28].

\section{Theory and Hypotheses}

In recent years there has been increasing discussion on the role of analytics in improving healthcare delivery [20]. Part of the reason that analytics is receiving more attention is that the technology that collects and stores clinical data is becoming more mature and is diffusing more rapidly [29]. Government mandates and incentives are also increasing uptake: for example, the HITECH Act [30] now requires that EHRs be used by all healthcare providers [31]. Even though there is a wide variety of EHR vendors, what is common across all of them is the ability to collect and store patient data in a standardized way. With these rich repositories of data emerging, analytics can be used in more meaningful ways, not the least of which is to demonstrate improved clinical decision making. In addition, the emergence of accountable care organizations (ACOs) in recent years has drawn even more attention to the value proposition of data analytics. ACOs require hospitals, physician groups, and other caregivers to come together and offer services that meet quality goals at a lower cost, tying provider reimbursement to quality metrics. The collaborative nature of ACOs requires that analytics be deployed to help the organization use resources "as efficiently as possible in a population perspective" [32, p. 7].

Despite significant potential value from these investments, several factors suggest that investment in HA may provide only some of its total potential value, and what value is reaped may be nuanced or heterogeneous. First, recent reports on the adoption of 
analytics in healthcare note that hospitals are still in the early stages of using clinical analytics and very limited information exists regarding the actual use of technologies that provide these capabilities [32, 33]. In particular, while long-term value in HA investments may lie in advanced data-driven prediction and decision making that meaningfully augment highly complex clinical care, most hospitals are currently focused on capabilities revolving around simply viewing and reporting on their existing data. One study found that $83 \%$ of providers report looking for analytics tools that "organize, analyze, and visualize" clinical, as well as financial and operational data [34]. That study also notes that most providers are seeking analytics vendors with "tools that provide clinical quality reporting."

Second, providers have been skeptical about the evolving role of analytics capabilities in healthcare, even with the existing functionality offered by current tools. For instance, some clinicians and researchers argue that "human intuition ... shouldn't be underrated" in clinical decision making and that "a computer may become a second opinion" but would not supplant clinical decision making in most contexts [35]. Similarly, others argue that "both the simplest tasks and the most complicated ones require people" and "humans are still superior at working with, and caring for others humans" as well as for grasping nuance and uncertainty [36]. A recent study found evidence of "algorithm aversion” where humans have a strong preference for a human decision maker, even when the human is objectively less accurate than an information system [21]. The study found that people lose confidence more quickly in an algorithm relative to a human after seeing them make the same mistake.

This understanding of the current state of healthcare analytics capabilities and use guides our subsequent theoretical development, which focuses on how HA capabilities can impact patient satisfaction and what factors moderate this effect.

\subsection{Theoretical Model}

The fundamental premise of OIPT is that the goal in organizational design is for firms to process information to reduce uncertainty [2]. Uncertainty can be defined as the difference between the amount of information required to perform the task and the amount of information already possessed by the organization [2, p. 36-37]. OIPT proposes that mechanisms such as standardized procedures and hierarchical referrals are appropriate for low-uncertainty environments, whereas computerized information systems and lateral relations are more appropriate for high-uncertainty environments. Subsequent refinements to OIPT proposed that firms process information to reduce not only uncertainty but also equivocality [1]. In contrast to uncertainty, which materializes from a lack of information, equivocality is driven by ambiguity rather than insufficient information [1]. Equivocality primarily occurs because of a lack of understanding caused by ambiguity, absence of problem structure, and little to no precedent for a problem [37].

Applying OIPT to the context of healthcare delivery, healthcare providers can improve patient outcomes if they process information in ways that reduce uncertainty and equivocality in clinical settings $[2,28]$. However, literature also suggests that reducing equivocality in clinical settings may require significantly richer information processing and much more meaningful use of that information relative to what is required for reducing uncertainty $[1,19]$. For instance, scholars suggest that "certain types of information processing mechanisms (such as computerized information systems and, by implication, systems with integrated data) provide large amounts of information and can thus help reduce uncertainty. However they are not as rich a source as other information processing mechanisms (such as face-toface meetings) and thus are not as effective in reducing equivocality” [19, p. 298].

Taking into account our prior discussion of the current state of HA adoption and how these tools are likely to be used, we extend this line of argumentation to HA and suggest that current capabilities of HA centered on the synthesis and aggregation of diverse and complex information would be well suited for reducing uncertainty in clinical (and similar) settings, but less adept at reducing equivocality. For example, although there are instances where IBM's Watson (a state-of-the-art natural language processing and analytics tool) helps reduce equivocality by diagnosing rare or perplexing medical conditions, thereby attracting interest from popular press [38], in practice the tool is much more commonly used to reduce uncertainty in terms of what treatments are safest and effective for a particular patient with an already known diagnosis [39, 40]. Moreover, uses of HA that augment existing processes to reduce uncertainty may be less threatening to physicians' autonomy and require less trust compared to more involved uses of HA that reduce equivocality (e.g., correcting a misdiagnosis by physicians), making gains from reduced uncertainty more likely to be realized. This suggests that even if complex analytics capabilities are available, phenomena like the "algorithm aversion" described previously may result in limited use of the tool to actually reduce equivocality [21].

We conjecture that in healthcare environments generally replete with decision uncertainty $[18,27]$, HA will improve patient satisfaction, on average, because 
of its significant potential to process information and resolve uncertainty. However, because HA is more likely to be effective at reducing uncertainty relative to equivocality, the effect of HA is argued to emerge unevenly across different clinical settings. This is because the uncertainty reductions that HA offers may be less valuable in clinical settings that also require the resolution of equivocality to achieve improvements in patient satisfaction.

We argue that two factors (clinical complexity and hospital concentration) moderate the extent to which resolution of equivocality (as opposed to uncertainty) is key to achieving gains in patient satisfaction. Our outcome is a multi-dimensional patient satisfaction measure and we present our research model in Figure 1.

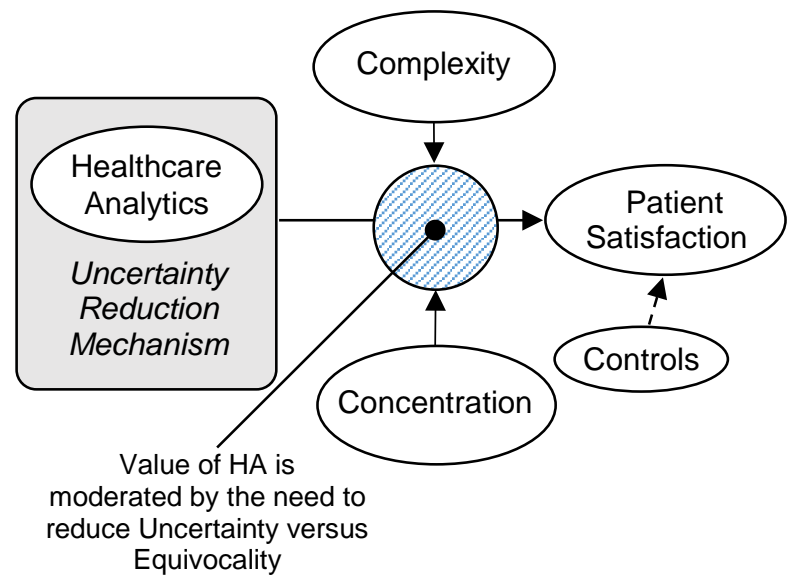

Figure 1. Research model

\subsection{Impact of healthcare analytics on patient satisfaction}

We propose a beneficial main effect of HA investments on patient satisfaction. Using the theoretical lens of OIPT, the primary mechanism through which this happens is the reduction of information uncertainty. According to OIPT, when there is a high degree of information uncertainty, one way organizations deal with this is by increasing the capacity to process information through investment in computerized information systems [19]. Healthcare analytics systems are one form of information system that can substantially contribute to uncertainty reduction because of their capabilities in data gathering and analysis, which ultimately can influence patient outcomes. These benefits may be substantial in a hectic and dynamic healthcare environment where uncertainty in decision making can emerge in many of the processes and activities necessary to the provision of effective clinical care $[17,18]$. Prior work has asserted that uncertainty is possibly the most important single influence on physician behavior [18, 27]. This work highlights a variety of sources of uncertainty in the delivery of healthcare, including the lack of information on the probabilities of treatment outcomes (even under controlled circumstances) and a disconnect between the utility of the physician-who makes vicarious decisions - and patient's utility [17, p. 517]. Thus, our contention is that HA will reduce uncertainty and offer value in many ways including but not limited to reductions in medical errors, improved coordination of care, better decision making, and improved communication.

Because extant research on the effect of HA is limited, we also interviewed several clinicians and senior administrators at hospitals and health systems. These informants conveyed a number of powerful anecdotes about HA and how its use reduces uncertainty and contributes to patient satisfaction. One respondent noted that the use of clinical analytics has contributed to his hospital's understanding of clinical processes and where bottlenecks occur, allowing the staff to better coordinate care for patients. This improved coordination of care has enabled them to dramatically reduce wait times of patients prior to being seen by a clinician and after the first encounter, which he noted is a key factor in patient satisfaction. Another interviewee from a very large health system said they use a population health management tool to look at 'big data' and synthesize it to identify at-risk subgroups. Then they attempt to engage these subgroups with targeted wellness approaches. We highlight these as instances where HA is used primarily to reduce uncertainty in clinical settings, whether it be around identifying processes that are inefficient or with respect to how to best tailor treatment for individual patients. These reductions in uncertainty in clinical settings should result in positive impacts, on average, on patient satisfaction. Therefore, we hypothesize that:

H1: The use of healthcare analytics will be positively related to patient satisfaction.

\subsection{Moderating effect of complexity and concentration}

We will present arguments suggesting that hospitals that treat low-complexity patients or are highly diversified will benefit from HA adoption since they can achieve substantial gains in patient satisfaction largely by reducing uncertainty. In contrast, hospitals that treat highly complex patients and/or are clinically specialized (i.e., high concentration) also require resolution of equivocality to achieve improved patient satisfaction, diminishing the benefit these hospitals receive from the adoption of HA. 
2.3.1. Complexity. Complexity in managing healthcare arises from the range and severity of the patient population catered to by the hospital. This is operationalized using the industry-standard case mix index (CMI) [41], with higher CMI indicating more complex cases. Although there exists significant variation between hospitals in the complexity and nature of patients they see [42], no study has examined the moderating role of complexity as it relates to the use of analytics in healthcare. We examine this question and contend that the complexity of the patients treated in a hospital may be an important moderator of realizing value from HA capabilities.

Case complexity ranges on a continuum: on one end of the spectrum are hospitals that generally treat lowcomplexity patients who are characterized by fewer comorbidities and less severe medical conditions. Care for these patients is less ambiguous since providers may be confident of underlying causes of the patients' symptoms and what general procedures are needed to properly treat the patient. In these instances, decisions and procedures that might be critical to patient satisfaction are more systematic and predictable, suggesting that equivocality is not a critical factor in driving improved patient satisfaction. This does not mean that the care of lower complexity patients is trivial; in fact, OIPT suggests that these types of decisions and procedures can benefit significantly from reductions in information uncertainty [19].

As hospitals move along the continuum towards the treatment of higher complexity patients, the value of reduction in uncertainty becomes less pronounced and resolution of equivocality matters more. Highcomplexity patients often have multiple medical conditions, rare diseases, and/or difficult-to-treat conditions, requiring providers to focus on reducing equivocality to deliver high quality care. To illustrate, consider an alternative scenario where a provider interacts with a patient with a set of symptoms that are not definitive of any specific condition, leaving the provider unsure about the steps to take to both diagnose and treat the patient. In this case, information processing mechanisms (potentially non-IS mechanisms) that reduce equivocality become critical to providing the best care for the patient. OIPT would suggest that consultation with other providers and specialists might be particularly useful in these cases [19]. Consistent with other work [19], if the equivocality in this case cannot be addressed, any information processing capabilities that reduce uncertainty will be of less value. For example, providers may try to treat patients for a variety of things simultaneously, limiting the value of HA that, for example, provides deep insights into tailored treatment when the cause is clear. Overall, this suggests that as hospitals move towards treating higher complexity patients, reducing equivocality, not uncertainty, will be most critical for improving patient satisfaction-but HA is not well suited to reducing equivocality. Drawing on our previous arguments that HA capabilities are likely to be highly valuable when uncertainty rather than equivocality is a key challenge, this suggests that hospitals that deal mostly in lowcomplexity patients would reap more value from HA investments relative to hospitals that deal in higher complexity patients. Formally, we hypothesize:

H2: Because of the uncertainty reduction capabilities of HA, the effect of HA on patient satisfaction will be greater in lower complexity hospitals.

2.3.2. Concentration. Another factor we consider is concentration-or more generally, the concept of organizational 'focus'-which refers to the allocation of hospital resources toward specific offerings of services [43, 44]. Low concentration signifies diversification of functions performed, while a high level of concentration indicates that the majority of resources are focused on only a small number of functions [43]. In the healthcare setting, higher concentration indicates that the hospital allocates a significant portion of its resources to a smaller number of clinical areas. For example, some hospitals are very specialized with the majority of their care efforts focused on a specific clinical context (e.g., cancer, heart disease, women's or children's care), while others are more general and treat a wide range of patients and conditions. Similar to complexity, we argue that concentration impacts the extent to which reducing uncertainty or equivocality matters for improved patient outcomes and satisfaction.

We first consider hospitals that are concentrated and focus on a specific set of clinical areas. Highly concentrated hospitals are likely to be marked by high population homogeneity and a deep institutional knowledge in their specific area of focus; this includes specialized experience of their providers as well as a robust understanding of processes to efficiently provide their specific type of care. Their deep knowledge and a more homogenous patient population suggest that uncertainty may be less of a factor in driving improved patient outcomes and satisfaction for these hospitals. On the other hand, equivocality may be a major factor for these hospitals. In particular, the patients they see may have very specific needs, suggesting that they would commonly have to deal with less structured, ambiguous care. Relatedly, providers in specialized hospitals may be experts in a particular clinical area, leading them to either trust their own experience and expertise over HA tools or to perceive existing tools as too rudimentary to be of use to them. Overall, we 
contend that achieving gains in patient satisfaction for highly concentrated hospitals depends more on resolution of equivocality than uncertainty.

As we move down this continuum towards hospitals that are more diverse in their focus (i.e., less concentrated), the need to reduce equivocality versus uncertainty starts to shift. Unlike highly concentrated hospitals, hospitals that split their focus across a number of care areas are less likely to develop deep knowledge or competence in any specific area of care. Without deep institutional investment into standard processes of care or tailored resources for each of their clinical areas, it becomes more likely that clinicians (even those specializing in that area of care) will face more uncertainty in the daily provision of care. For instance, compared to a pediatrician at a children's hospital, a pediatrician at a diversified hospital may have fewer colleagues to draw insights from, less institutional knowledge to support their activities, and less training to handle various clinical needs of even patients who require standard care (i.e., when equivocality is low). At the same time, resolution of equivocality may be less important for improving patient satisfaction in diversified hospitals. Equivocality may simply be lower since patients with highly unstructured care needs may seek concentrated facilities that can address their specific needs better. For these reasons, we contend that reducing uncertainty in diverse hospitals (i.e., low in concentration) will result in substantial gains in patient satisfaction. Leaning on our previous arguments that HA capabilities are likely to be highly valuable when uncertainty is a key challenge, this suggests that hospitals that are high in concentration would reap less value from HA investments relative to hospitals that are more diverse. Formally, we hypothesize:

$\underline{H 3}$ : Because of the uncertainty reduction capabilities of $H A$, the effect of HA on patient satisfaction will be greater in low concentration hospitals.

\section{Data}

Our empirical approach leverages an eight-year panel dataset (2007-2014). Our central outcome measure of patient satisfaction comes from the Hospital Consumer Assessment of Healthcare Providers and Systems (HCAHPS) survey on patient satisfaction. These data are collected annually for the vast majority of hospitals in the U.S. and include ten questions capturing patient perspectives on care across several topics: communication with doctors, communication with nurses, responsiveness of hospital staff, pain management, communication about medicines, discharge information, cleanliness of the hospital environment, and quietness of the hospital environment, an overall hospital rating and if they would recommend the hospital. All of these dimensions are measured on a scale of $0-100$. We take the average of patients' assessments across all ten of these dimensions for a given hospital to generate our annual measure of patient satisfaction. To identify hospital analytics capabilities, we leverage data from the annual HIMSS Analytics Database (HADB) which captures various dimensions of hospital technology capabilities. Specifically, we focus on hospital reports of investment in "Business Intelligence" or "Data Mining” and designate a hospital as having Clinical Healthcare Analytics (HA) capabilities if it reported investing in clinically focused business intelligence and data mining.

We also leverage diverse datasets to measure the factors that may moderate the impact of analytics capabilities on patient satisfaction: Complexity and Concentration. Complexity is measured using annual data from the Centers for Medicare and Medicaid Services (CMS) on a given hospital's CMI, which reflects the mix of patients in higher versus lower severity diagnosis-related groups (DRGs) [41].

We measure our other moderator, Concentration, by drawing upon prior work [43], which calculates a Herfindahl-Hirschman Index (HHI) for a given hospital that captures whether a hospital has a narrow or widely varied clinical focus. Specifically, we leverage Hospital Compare data to identify the number of medical cases in the hospital across ten Major Diagnostic Categories (MDC), based on the 70 most common DRGs as designated by CMS. Following the method for computing the HHI [43], we first tally the total number

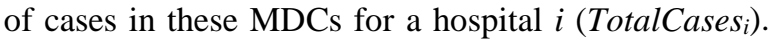
Then we calculate the proportion that any one MDC $j$ accounted for relative to the total cases. These values are then squared and summed to generate a value between 0 and 1. Hospitals with higher values of HHIConc represent those that focus on one or a few clinical areas, whereas hospitals with lower values offer a diverse set of clinical areas.

$$
\text { HHIConc }_{\mathrm{i}}=\sum_{j=1}^{n}\left(\frac{\text { CasesInMDC }_{i j}}{\text { TotalCases }_{i}}\right)^{2}
$$

Finally, we leverage the HADB to identify a number of relevant hospital controls as noted in Table 1 . The final dataset has 14,677 observations and includes data on 3,126 hospitals. By 2014, 891 of these hospitals ( $29 \%$ ) reported adopting HA.

$$
\text { MarketComp }_{i}=\sum_{i=1}^{n}\left(\frac{\text { StaffedBeds }_{i}}{\text { TotalStaffedBeds }_{k}}\right)^{2}
$$




\section{Main Estimation and Results}

We estimate the various relationships in our model using econometric models for panel data. Specifically, we estimate an OLS, panel fixed effects model including both time and hospital fixed effects and robust standard errors. Our main specification is:

PatientSatisfaction $_{i t}=\beta_{1} *$ HAClinical $_{i t}+$ $\gamma^{*}$ CONTROLS $i t+\theta_{i}+\lambda_{t}+\mu_{i t}$

PatientSatisfaction $_{i t}$ is the average patient satisfaction for hospital $j$ at time $t$. HAClinical it is a dichotomous variable indicating whether a hospital $i$ at time $t$ reported having adopted clinically focused data analytics capabilities. In our main estimation, we treat this adoption decision as binary but our results are robust to the relaxation of this assumption. In addition to our main variables of interest, we include a vector of controls to account for other relevant observables, potentially associated with patient satisfaction and a hospital's choice to pursue analytics capabilities. First, we include a control for the adoption of other health IT that may influence patient satisfaction and also correlate with analytics capabilities (EHRAdoption ${ }_{i t}$ and Portals $_{i t}$ ). We also include a number of controls focused on hospital characteristics. Finally, we include a measure of market competition using the HHI index for hospitals in a hospital referral region based on hospital staffed beds. This control is useful if highly competitive markets care more about patient satisfaction and are also more likely to pursue clinical analytics capabilities.

We also include hospital and time fixed effects (represented by $\theta_{\mathrm{i}}$ and $\lambda_{\mathrm{t}}$, respectively); $\mu_{\mathrm{it}}$ is the error term. Hospital fixed effects allow us to control for timeinvariant factors that could simultaneously drive the emergence of analytics capabilities and changes in patient satisfaction. For instance, it may be the case that academic hospitals are more likely to invest in analytics and also have more of a focus on patient-centric care. Because healthcare markets do not change very quickly over time, hospital fixed effects are a key dimension of identification in our estimated model. Time fixed effects allow us to control for time trends in our data and any shocks that impact all hospitals in a given year; for example, any legislation that applies nationally would be captured by our time fixed effects.

In an extension of our main model, we estimate the impact of our two main moderating variables by interacting them with our measure of HA capabilities. Specifically, we consider our first moderating factor, Complexity, captured by the case mix index of hospital

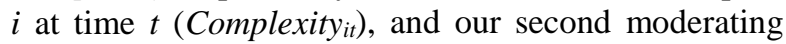
factor, Concentration, captured by the hospital-specific HHI derived from its mix of clinical activities $\left(H H I C o n c_{i t}\right)$. This extended model allows us to tease out any differential effects of HA under different levels of patient complexity and hospital concentration.

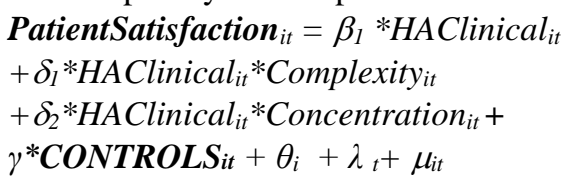

\subsection{Results}

We first evaluate the main effect of HA on patient satisfaction (hypothesis 1) using a random effects model (Table 1, column 1), which identifies initial evidence of a positive impact of $\mathrm{HA}$ on patient satisfaction $\left(\beta_{\text {HAClinical }}=.468, \mathrm{p}<.01\right)$. We then estimate a fixed effects model which replicates our random effects model with the addition of hospital fixed effects that account for time-invariant hospital factors that may bias our estimation (column 2). We find consistent results with our random effects estimation ( $\beta_{\text {HAClinical }}$ $=.410, \mathrm{p}<.01$ ), confirming evidence for a positive main effect of clinical analytics capabilities on patient satisfaction, providing support for $\mathrm{H} 1$. This positive and significant result speaks to HA's capabilities, on average, to positively impact hospital processes and activities related to patient satisfaction.

Table 1. Main results

\begin{tabular}{|c|c|c|c|}
\hline VARIABLES & $\begin{array}{l}\text { Random } \\
\text { Effects }\end{array}$ & $\begin{array}{c}\text { Fixed } \\
\text { Effects }\end{array}$ & $\begin{array}{c}\text { Full } \\
\text { Model }\end{array}$ \\
\hline HAClinical & $\begin{array}{c}0.468 * * * \\
(0.101)\end{array}$ & $\begin{array}{c}0.410 * * * \\
(0.104)\end{array}$ & $\begin{array}{c}2.608 * * * \\
(0.783)\end{array}$ \\
\hline $\begin{array}{l}\text { HAClinical* } \\
\text { Complexity }\end{array}$ & & & $\begin{array}{c}-0.902 * * * \\
(0.340)\end{array}$ \\
\hline $\begin{array}{l}\text { HAClinical* } \\
\text { HHIConc }\end{array}$ & & & $\begin{array}{l}-2.414 \\
(1.618)\end{array}$ \\
\hline EHRAdoption & $\begin{array}{l}0.0425^{*} \\
(0.0219)\end{array}$ & $\begin{array}{c}0.0494 * * \\
(0.0221)\end{array}$ & $\begin{array}{c}0.0496 * * \\
(0.0221)\end{array}$ \\
\hline Portals & $\begin{array}{c}0.259 * * * \\
(0.0800)\end{array}$ & $\begin{array}{c}0.247 * * * \\
(0.0824)\end{array}$ & $\begin{array}{c}0.261 * * * \\
(0.0825)\end{array}$ \\
\hline NoStaffedBeds & $\begin{array}{c}-0.0073^{* * *} \\
(0.000650)\end{array}$ & $\begin{array}{l}-0.00121 \\
(0.00118)\end{array}$ & $\begin{array}{l}-0.00106 \\
(0.00120)\end{array}$ \\
\hline NoOutVisits & $\begin{array}{c}-1.30 \mathrm{e}-07 \\
(1.75 \mathrm{e}-07)\end{array}$ & $\begin{array}{c}-1.33 \mathrm{e}-07 \\
(1.80 \mathrm{e}-07)\end{array}$ & $\begin{array}{c}-1.15 \mathrm{e}-07 \\
(1.84 \mathrm{e}-07)\end{array}$ \\
\hline NoERVisits & $\begin{array}{c}-5.4 \mathrm{e}-07 * * * \\
(1.73 \mathrm{e}-07)\end{array}$ & $\begin{array}{c}-2.07 \mathrm{e}-07 * * * \\
(7.54 \mathrm{e}-08)\end{array}$ & $\begin{array}{c}-2.1 \mathrm{e}-07 * * * \\
(7.49 \mathrm{e}-08)\end{array}$ \\
\hline NoOperatRooms & $\begin{array}{c}0.0329 * * * \\
(0.0103)\end{array}$ & $\begin{array}{c}0.0163 \\
(0.0106)\end{array}$ & $\begin{array}{c}0.0175 \\
(0.0107)\end{array}$ \\
\hline NoPatientDays & $\begin{array}{c}1.67 \mathrm{e}-09 * \\
(9.61 \mathrm{e}-10)\end{array}$ & $\begin{array}{l}1.07 e-09 * \\
(6.48 e-10)\end{array}$ & $\begin{array}{c}9.84 \mathrm{e}-10 \\
(6.50 \mathrm{e}-10)\end{array}$ \\
\hline Complexity & $\begin{array}{c}1.241 * * * \\
(0.261)\end{array}$ & $\begin{array}{l}0.618 * \\
(0.352)\end{array}$ & $\begin{array}{l}0.622 * \\
(0.355)\end{array}$ \\
\hline MarketCompet & $\begin{array}{c}3.391 * * * \\
(0.675)\end{array}$ & $\begin{array}{l}-1.640 \\
(1.441)\end{array}$ & $\begin{array}{l}-1.635 \\
(1.442)\end{array}$ \\
\hline Constant & $\begin{array}{c}70.14 * * * \\
(0.395)\end{array}$ & $\begin{array}{c}70.74 * * * \\
(0.643)\end{array}$ & $\begin{array}{c}70.67 * * * \\
(0.655)\end{array}$ \\
\hline $\mathrm{R}^{2}$ & .315 & .320 & .323 \\
\hline Observations & 14,677 & 14,677 & 14,542 \\
\hline HOSPITAL FE & $\mathrm{NO}$ & YES & YES \\
\hline YEAR FE & YES & YES & YES \\
\hline
\end{tabular}


With evidence of a main effect of HA on patient satisfaction, we turn to the potential moderating role of hospital complexity and concentration (column 3). Estimating our full model, we find that the largest gains from clinical analytics capabilities accrue to hospitals with low clinical complexity but broad clinical concentration. Specifically, we see that these gains diminish as clinical complexity increases, with a large and significant coefficient on the interaction of HAClinical and Complexity $\left(\beta_{\text {HAClinical }}{ }^{*}\right.$ Complexity $=-0.902$, $\mathrm{p}<.001-\mathrm{H} 2$ supported) and a directionally consistent (but insignificant) coefficient on the interaction between HAClinical and Concentration $\left(\beta_{\text {HAClinical }{ }_{\text {HHIConc }}=-}\right.$ 2.414, not significant-H3 not supported). Overall, our results suggest that hospitals at the lower end of the complexity spectrum may see as much as a 2-point gain in patient satisfaction from analytics implementation. Yet, it is surprising that concentration did not moderate the relationship. As noted in our theoretical justification, there were ex ante reasons to believe that more diversified hospitals, which are more siloed and have less depth in any given field of care, might benefit more from uncertainty reductions and thus reap more benefit from analytics. In an effort to better understand the nonsignificant result for concentration, we partitioned our analysis by large and small hospitals (using a median split). We conjectured that the factors that drive information uncertainty in diversified hospitals may be more pronounced in larger hospitals because information silos may be pronounced. We reanalyzed the data and found a moderating effect of concentration is significant and negative in large hospitals (table removed due to space constraints). We also conducted robustness checks and performed endogeneity checks that were removed to conserve space.

\section{Discussion}

We evaluate the effect of healthcare analytics following guidance provided through the application of OIPT and find a significant main effect of analytics on patient satisfaction as well as meaningful evidence of a theoretically relevant moderator of this effect. At the same time, we acknowledge that our study has some important limitations. Our measure of clinical analytics is admittedly coarse, but we do find our results to be robust to a variety of conditions and our dataset is extremely large relative to the population of hospitals. Moreover, this problem is not unique to analytics as the same issue existed in early studies of EHRs where only three or fewer technologies made up a suite [29].

Another limitation is that we argue that OIPT provides the theoretical mechanism through which HA acts on patient satisfaction and that complexity and concentration have a moderating effect on this relationship. Since we do not directly measure information processing, we cannot conclude that it is the only theoretical mechanism that could be used to inform the relationships we observe. We believe our argumentation to be compelling, but there is a chance that other theoretical frameworks could similarly inform the value of analytics capabilities in healthcare settings. These limitations are largely due to data constraints, which we hope to address in future works by collecting primary data on actual information processing capacity in a specific clinical setting.

We first discuss the main effect of HA on patient satisfaction, which yielded a regression coefficient of 0.468 . While small, there is reason to be optimistic about this magnitude. First, the effect size increases by more than two points (regression coefficient of 2.608 in Table 1, column 3) when considering the moderating effects of complexity and concentration. Second, industry observers note that the cost of low patient satisfaction scores is non-trivial and it is extremely difficult to overcome perceptions of poor care [45, 46]. Now that CMS provides reimbursements based on patient satisfaction scores, this has become an even greater strategic issue for hospitals [46].

Our results confirm our second hypothesis that hospitals with high-complexity cases may see diminished gains from analytics capabilities, at least in terms of patient satisfaction. We argue that this may occur due to the equivocality that results from treating highly complex patients. It is more likely that hospitals are seeking information about general trends or simply using basic features of HA that provide elementary information rather than condition-specific complex data about complicated cases. This would explain why lower complexity hospitals have greater gains from HA. But it does highlight a more general and critical point. As analytics capabilities evolve to become more robust, it is possible, and maybe even likely, that value will increase for higher complexity cases in the long term. As a result, we think it is important for future researchers to investigate whether the effect of complexity may differ as analytics capabilities mature.

In hindsight it is not surprising that we did not find a significant effect of concentration in the full population of hospitals. To the extent that the value from analytics grows exponentially with the amount of data available, it is expected that it would be larger hospitals that reap the most benefits, at least in the short term. Over time, as smaller hospitals accrue larger data stores, they are likely to benefit as well. It is important to recognize that analytics capabilities are still regarded as new and researchers typically do not have access to actual use data. Until these data become available, it is important to take preliminary steps toward 
understanding the value proposition of data analytics in general, but it is possible that we are expecting 'too much too soon' in terms of understanding the complex dynamics associated with integrating analytics into long-established workflows.

\section{Conclusion}

Our study is a first attempt at theorizing how hospitals use analytics to reap benefits. The theoretical frame of OIPT provides the basis for our argument that $\mathrm{HA}$ is an uncertainty-reducing mechanism that impacts patient satisfaction. We proposed a nuanced moderating role of complexity of patient cases and concentration and while the conditional effect of concentration is somewhat unclear, the main effect of HA on satisfaction is unambiguously positive-suggesting that analytics is reducing uncertainty with healthcare delivery tasks and improving patient satisfaction. Also, the conditional effect of complexity on value from analytics seems defensibly negative, presumably via its theorized impact of increasing uncertainty relative to the current state of analytics capabilities. The findings are important first steps toward investigating how value can be extracted from data analytics.

\section{References}

[1] Daft, R.L. and R.H. Lengel, Organizational Information Requirements, Media Richness and Structural Design. Management Science, 1986. 32(5): p. 554-571.

[2] Galbraith, J.R., Organization Design. 1977, Reading, MA: Addison-Wesley.

[3] Orlikowski, W.J. and C.S. Iacono, Research Commentary: Desperately Seeking the "IT" in IT Research-A Call to Theorizing the IT Artifact. Information Systems Research, 2001. 12(2): p. 121-134.

[4] Jernigan, S., S. Ransbotham, and D. Kiron, Data Sharing and Analytics Drive Success with IoT: Creating business value with the Internet of Things. MIT Sloan Management Review, 2016. 587(1): p. 1-16.

[5] Ransbotham, S., D. Kiron, and P.K. Prentice, Beyond the Hype: The Hard Work Behind Analytics Success. MIT Sloan Management Review, 2016. 57(3): p. 1-16.

[6] Brynjolfsson, E., The Productivity Paradox of Information Technology. Communications of the ACM, 1993. 36(12): p. 66-77.

[7] Devaraj, S. and R. Kohli, Performance Impacts of Information Technology: Is actual usage the missing link? Management Science, 2003. 49(3): p. 273-299.
[8] Devaraj, S. and R. Kohli, Information Technology Payoff in the Healthcare Industry: A longitudinal study. Journal of Management Information Systems, 2000. 16(4): p. 41-67.

[9] McCullough, J.S., S. Parente, and R. Town, Health Information Technology and Patient Outcomes: The role of organizational and informational complementarities, in NBER Working Paper Series. 2013, National Bureau of Economic Research: Cambridge, MA. p. 1-45.

[10] Dutta, A., H.L. Lee, and S. Whang, RFID and Operations Management: Technology, value, and incentives. Production and Operations Management, 2007. 16(5): p. 646-655.

[11] Hitt, L.M. and E. Brynjolfsson, Information Technology and Internal Firm Organization: An Exploratory Analysis. Journal of Management Information Systems, 1997. 14(2): p. 81-101.

[12] Menon, N.M., U. Yaylacicegi, and A. Cezar, Differential Effects of the Two Types of Information Systems: A hospitalbased study. Journal of Management Information Systems, 2009. 26(1): p. 297-316.

[13] Angst, C.M., S. Devaraj, and J. D'Arcy, Dual Role of ITAssisted Communication in Patient Care: A Validated Structure-Process-Outcome Framework. Journal of Management Information Systems, 2012. 29(2): p. 255-291.

[14] Angst, C.M., et al., Performance Effects Related to the Sequence of Integration of Healthcare Technologies. Production and Operations Management, 2011. 20(3): p. 319333.

[15] Bardhan, I.R. and M.F. Thouin, Health Information Technology and its Impact on the Quality and Cost of Healthcare Delivery. Decision Support Systems, 2013. 55(2): p. 438-449.

[16] Versel, N. Cleveland Clinic, Other Health Groups Use Data to Boost Patient Satisfaction. U.S. News 2015 [cited 2015 June 10]; Available from: http://tinyurl.com/Versel2015.

[17] Wennberg, J.E., Commentary: On patient need, equity, supplier-induced demand, and the need to assess the outcome of common medical practices. Medical Care, 1985. 23(5): p. 512-520.

[18] Balsa, A.I., et al., Clinical Uncertainty and Healthcare Disparities. American Journal of Law \& Medicine, 2003. 29: p. 203-219.

[19] Goodhue, D.L., M.D. Wybo, and L.J. Kirsch, The Impact of Data Integration on the Costs and Benefits of Information Systems. MIS Quarterly, 1992. 16(3): p. 293-311. 
[20] Murdoch, T.B. and A.S. Detsky, The Inevitable Application of Big Data to Health Care. Journal of the American Medical Association, 2013. 309(13): p. 1351-1352.

[21] Dietvorst, B.J., J.P. Simmons, and C. Massey, Algorithm Aversion: People erroneously avoid algorithms after seeing them err. Journal of Experimental Psychology: General, 2015. 144(1): p. 114-126.

[22] Agha, L., The Effects of Health Information Technology on the Costs and Quality of Medical Care. Journal of Health Economics, 2014. 34(March): p. 19-30.

[23] Buntin, M.B., et al., The Benefits Of Health Information Technology: A Review Of The Recent Literature Shows Predominantly Positive Results. Health Affairs, 2011. 30(3): p. $464-471$.

[24] Queenan, C., C.M. Angst, and S. Devaraj, Doctors' Orders - If they're electronic, do they improve patient satisfaction? A complements/substitutes perspective. Journal of Operations Management, 2011. 29(7-8): p. 639-649.

[25] Devaraj, S., L. Krajewski, and J.C. Wei, Impact of eBusiness Technologies on Operational Performance: The role of production information integration in the supply chain. Journal of Operations Management, 2007. 25(6): p. 11991216.

[26] Agarwal, R. and V. Dhar, Editorial-Big Data, Data Science, and Analytics: The opportunity and challenge for IS research. Information Systems Research, 2014. 25(3): p. 443448.

[27] Galbraith, J.R., Organization Design: An information processing view. Interfaces, 1974. 4(3): p. 28-36.

[28] Flynn, B.B. and E.J. Flynn, Information-processing alternatives for coping with manufacturing environment complexity. Decision Sciences, 1999. 30(4): p. 1021-1052.

[29] Angst, C.M., et al., Social Contagion and Information Technology Diffusion: The Adoption of Electronic Medical Records in US Hospitals. Management Science, 2010. 56(8): p. 1219-1241.

[30] Freedman, L.F. and N. Peabody, The Health Information Technology for Economic and Clinical Health Act (HITECH Act): Implications for the adoption of health information technology, HIPAA, and privacy and security issues. 2009, Nixon Peabody. p. 1-4.

[31] Blumenthal, D., Launching HITECH. New England Journal of Medicine, 2010. 362(5): p. 382-385.

[32] Terry, K., Analytics: The nervous system of IT-enabled healthcare - Strategies for managing sophisticated analytic tools in the health care industry. 2013, New York, NY: Institute for Health Technology Transformation. 1-24.

[33] Dergurahian, J., Analytics: Moving health care forward. 2011, SearchHealthIT.com: Newton, MA. p. 1-9.

[34] Graaff, J.V.D. and A. Cameron, Business Intelligence Perception 2012: A wave is coming. 2012, KLAS.

[35] Chan, L. Will Robots In Healthcare Make Doctors Obsolete? 2016 [cited 2016 August 1]; Available from: http://tinyurl.com/ChanRobots2016.

[36] Mesko, B., My Health: Upgraded: Revolutionary Technologies To Bring A Healthier Future. 2015, Budapest, Hungary: Webicina Kft.

[37] Daft, R.L., R.H. Lengel, and L.K. Trevino, Message Equivocality, Media Selection, and Manager Performance: Implications for information systems. MIS Quarterly, 1987. 11(3): p. 355-366.

[38] Ng, A. IBM's Watson Gives Proper Diagnosis for Japanese Leukemia Patient after Doctors were Stumped for Months. 2016 [cited 2016 December 13]; Available from: http://tinyurl.com/NgIBM2016.

[39] Kohn, M.S., et al., IBM's Health Analytics and Clinical Decision Support. Yearbook of Medical Informatics, 2014. 9(1): p. 154-162.

[40] Hernandez, D. Artificial Intelligence is Now Telling Doctors How to Treat You. 2014 [cited 2016 December 11]; Available from: http://tinyurl.com/Wired2014AI.

[41] Fetter, R.B., et al., Case Mix Definition by DiagnosisRelated Groups. Medical Care, 1980. 18(2): p. 1-53.

[42] Steinwald, B. and L.A. Dummit, Hospital Case-Mix Change: Sicker patients or DRG creep? Health Affairs, 1989. 8(2): p. 35-47.

[43] Ding, D.X., The effect of experience, ownership and focus on productive efficiency: A longitudinal study of US hospitals. Journal of Operations Management, 2014. 32(1): p. $1-14$.

[44] Skinner, W., The Focused Factory. Harvard Business Review, 1974. 52(3): p. 113-121.

[45] Rau, J., Hundreds of Hospitals Struggle to Improve Patient Satisfaction. 2015, Kaiser Health News: Salisbury, NC. p. 1.

[46] Siegrist, R.B.J., Patient Satisfaction: History, myths, and misperceptions. AMA Journal of Ethics, 2013. 15(11): p. 982987. 